\title{
Éros énergumène de Denis Roche, une écriture de l'agapè à rebours de l'orgie thanatique
}

\author{
Denis Roche's Éros énergumène, a Writing of Agape Against \\ the Thanatic orgy
}

Gouhé Ouattara

Université Alassane Ouattara, Côte d'Ivoire

\begin{abstract}
Résumé : Évoquer l'écriture poétique consiste, le plus souvent, à faire resurgir à la mémoire une somme de conventions artistiques établies d'âge en âge à travers les genres, les courants et les conceptions de la poésie. Malgré les révolutions et les bouleversements intervenus, l'art poétique se départit difficilement des cloisons génériques et théoriques pour le moins étanches. Cependant, l'actualité de la littérature française révèle que certains textes de la seconde moitié du XXe siècle semblent accorder une sorte de " vacance » à la poésie afin qu'il lui soit loisible de s'investir davantage dans l'autonomie langagière. Éros énergumène de Denis Roche illustre fondamentalement cette aptitude de l'écriture poétique à « se voir » comme telle et à se dématérialiser dans une perspective, somme toute, spiritualiste. Le poème de Roche s'appréhende, de ce fait, comme une voie d'accès à une forme de jeu intellectuel où le lecteur averti se retrouve englouti finalement dans les tourbillons d'un langage qui n'a d'autre enjeu que la liberté absolument «libérée ».
\end{abstract}

Mots-clés : agapè, écriture poétique, éros, langage poétique, liberté, spiritualité.

Abstract: To evoke poetic writing is, more often than not, to bring to memory a sum of artistic conventions established from age to age through genres, currents and conceptions of poetry. Thus, in spite of the revolutions and upheavals that have taken place, the poetic art is difficult to separate from generic and theoretical partitions, at the very least, watertight. However, the topicality of French literature reveals that some texts of the second half of the $20^{\text {th }}$ century seem to grant a sort of "vacancy" to poetry, so that it may be more active in language autonomy. Denis Roche's Éros énergumène illustrates fundamentally this aptitude of poetic writing to "see oneself" as such and to dematerialize oneself in a perspective altogether spiritualist. Roche's poem can be understood as a way of accessing a form of intellectual play where the informed reader is finally engulfed in the whirlwinds of a language that has no other stake than an absolutely "liberated" freedom.

Keywords: agape, poetic writing, eros, poetic language, freedom, spirituality.

\section{Introduction}

Le langage poétique possède le plus souvent un caractère réflexif, en tant qu'il se consacre essentiellement à l'expression d'une sorte d'intériorité dans laquelle il semble se complaire. Dans ce cas, la poésie est faite pour elle-même, elle n'a d'autre but qu'elle-même, selon le vœu d'absolu des symbolistes', par exemple. Cela n'est pas possible sans une dose suffisante d'énigme et de mystère dans l'acte d'écrire du

\footnotetext{
1 Les initiateurs du symbolisme au XIXe siècle, en l'occurrence Rimbaud, Verlaine et Mallarmé sont susceptibles d'être évoqués à ce niveau.
} 
poète, qui dispose à la fois de son intimité et de tout autre matériau environnemental et événementiel. Mircea Eliade, voulant énoncer le caractère secret de l'inspiration poétique, affirme ceci :

Toute poésie est un effort pour recréer le langage, en d'autres termes pour abolir le langage courant, de tous les jours, et inventer un nouveau langage, personnel et privé, en dernière instance secret... Voilà qui rappelle étrangement le comportement du "primitif » et de l'homme des sociétés traditionnelles².

La conception traditionnelle confère ainsi au texte poétique une certaine "incommunicabilité " que Denis Roche prend en compte dans cette constatation: « Ainsi par la grâce d'une re-création, se trouve définitivement fixé un langage absolu, incommunicable, un no man's land infranchissable, mais qui autorise peut-être le survol $)^{3}$.

Ce "survol" évoqué par le poète avisé peut se concevoir comme le sentier à emprunter pour qu'une incursion véritable soit effectuée dans l'arène intellectuelle du texte poétique. Le terme éros apparaît, dans sa généralité sémantique, comme la manifestation de l'amour charnel; un tel amour s'actualise le plus souvent dans un contexte démentiel, où la luxure à outrance génère une sorte d'illogisme pouvant choquer la raison. À l'opposé ou en complément, se trouve l'amour spirituel qui est désincarné et qu'il convient de nommer agapè. À la lecture de la première phrase du résumé de l'ouvrage de Père Marcelo Rossi, la confirmation d'une forme "divine " d'éros est manifeste : « Agapè est le mot grec pour l'amour divin, inconditionnel et spirituel, s'ajoutant à Éros, l'amour physique, Storgé, l'amour familial, et Philia, l'amitié4. ") Cette acception, somme toute philosophique, laisse entrevoir l'idée que l'amour, conçu habituellement sous ses aspects érotique et thanatique, peut s'éthériser jusqu'à se confondre avec l'absolus.

L'œuvre poétique' de l'écrivain photographe Denis Roche est taxée constamment de "pragmatisme érotique $\|^{7}$ par bon nombre de critiques et théoriciens de la littérature, en raison même de l'occurrence abondante des symboles lexicaux liés aux ébats amoureux. Cependant, cette aptitude intellectuelle, consistant à considérer amplement l'image sexuelle derrière l'amoncèlement de mots et de signes textuels, peut donner à voir ou à lire également le motif d'une orgie spirituelle parfaitement intellectualisée. Tel est le cas dans Éros énergumène dont l'écriture fortement déséquilibrée autorise tout de même la présence évidente de repères de la

\footnotetext{
2 Eliade, M. (1989). Mythes, rêves et mystères. Paris : Gallimard, p. 36.

3 Roche, D. (1962). La Poésie est inadmissible. Paris : Sevil.

${ }^{4}$ Rossi, M. (2010). Agapè ; l'amour sans limites. Paris : Édition Robert Laffont.

5 Dans ce sens, il faut citer Françoise Frazier qui, dans un article intitulé « Eros et Philia dans la pensée et la littérature grecques », conclut, au sujet de l'amour platonicien, en des termes symbolistes : "L'analyse d'Eros s'élève ainsi à des sommets métaphysiques qui resteront l'apanage des platoniciens ». ("Eros et Philia dans la pensée et la littérature grecques. Quelques pistes, d'Homère à Plutarque ॥, dans la revue Vita Latina, 177, Université Montpellier III, 2007, pp. 31-44,

https://www.persee.fr/doc/vita_0042_7306_2007_num_177_1_1242 [19/02/2021].

6 Référence est faite, entre autres, à Forestière amazonide (1962), Les Idées centésimales de Miss Elanize (1964), Eros énergumène (1968), Dialogues du paradoxe et de la barre de mine (1968), Le Mécrit (1972). 7 L'expression est empruntée à Stéphane Baquey qui a intitulé l'un de ses articles en ces termes : "La poésie de Denis Roche. Proposition d'un protocole de lecture : une pragmatique érotique ", in Aletria, Belo Horizonte, 3, pp. 147-171, 2017. Dans le même sens, on pourrait aussi évoquer l'analyse de Bertrand Laverdure dans Spirale (novembre-décembre 2002) : «Poésie et perversité ».
} 
spiritualité. II s'avère donc impérieux de chercher à déceler l'intention du poète, qui intègre à l'écriture de la déraison amoureuse une saveur philosophique. Ainsi se formule l'objectif de cette analyse organisée autour de trois axes essentiels : l'écriture du délire érotique ; Éros comme un exposé de l'amour spirituel ; la démence réaliste à l'encontre du mythe surréaliste.

\title{
1. L'écriture du délire érotique
}

L'orgie (sexuelle) rochienne entre dans sa phase concrète par l'écriture qui, une fois submergée par le plaisir incontrôlé, l'actualise démesurément. Cette partie liminaire s'intéresse aux formes lexicales et imagées dont se sert Roche pour « lubrifier » le texte par jets saccadés et rythmés.

\subsection{Indices lexicaux de la sensualité}

Les premiers vers d'Éros énergumène donnent déjà le ton d'un étalement rythmé de lexèmes de la débauche. L'extrait de la page 26 confirme davantage cette impression :

\author{
Sa vengeance (mais c'est toujours cette suite) \\ L'attache miséracle de tout coït silencieux \\ Le carquois - le harnais - retombe, bruit, re- \\ Monte et retombe contre sa cuisse, bruit, sur \\ Celles, qu'il cite, notamment le trifolium \\ (Encore une fois ça lui fait mal, mais ces \\ Chocs ne sont pas du tout accordés au rythme que \\ Je prends pour le dire) encore un choc, avec \\ L'éclat souriant et bruyant propre à l'arrivée \\ En altitude. Les pentes, plus sèches, sont en \\ proie aux broussailles... très denses avec le \\ Timbre caractéristique, il y a aussi les cous- \\ Sinets de genévrier et l'abardal, comme ils \\ Disent, coriace et vénéneux. Moi je t'ai \\ Faite à l'image du paysage à cheval == pics \\ Couronnés de neiges que l'on voit sur les \\ Murs de la Maison Blanche, ou bien type Hall- \\ De-Grand-Bâtiment-Administratif, au Middle West.
}

À la lecture de cet extrait, l'impression de se trouver en présence d'une gigantesque et impitoyable scène orgiaque ("ça fait mal », « carquois »: " choc »), régentée par le « coït», est évidente. L'intensité de l'activité sexuelle se perçoit d'emblée à travers la mise en relief du mot « coït » au deuxième vers, et qui envahit ainsi l'ensemble du texte. Les lexèmes relatifs au coït, notamment " carquois » et « harnais " composent à première vue une grande toile faite d'images de lutte et de rut dans leur acception charnelle. À ces indices vient s'ajouter la répercussion sonore du phonème consonantique [k], initial de « coït» ( $\vee .2)$, au début, à l'intérieur ou à la fin de plusieurs mots : " carquois » (v. 3), " contre », « cuisse » (v. 4), "Chocs» (v. 7 et 8), " accordés» (v. 7), " encore » (v. 8), " avec » (v. 8 et 1 1), « caractéristique », « cous » (v. 12), " comme " (v. 13), « coriace » (v. 14), « pics» (v. 15), « Couronnés » (v. 16).

De toute évidence, chaque apparence du poème est soigneusement investie et innervée par le signifiant copulateur [k] renforcé par la présence du monème " qu(e) », donnant ainsi lieu à un grand concert sexualisé et sexuel. De plus, il s'opère 
dans le poème, à travers un rythme particulier agencé par les mots eux-mêmes, un spectacle visible d'animations amoureuses :

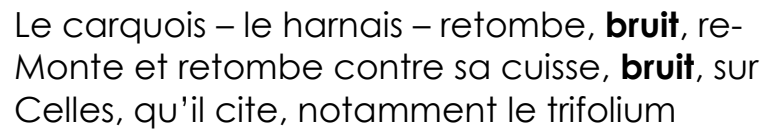

Avec l'apparition régulière de «bruit » (mis en parallèle) dans les deux premiers vers, Roche sollicite systématiquement l'ovïe du spectateur invité à entendre la chevauchée délirante. Chevauchée qu'il figure d'ailleurs aux vers 14 et 15 («Moi je †'ai / Faite à l'image du paysage à cheval ॥) et qui se concrétise par l'apparition de la gelée blanche libidinale, voire séminale, à travers l'image des « pics / Couronnés de neige $»$ (v. 16-17).

Il existe dès lors une sorte de violence du spectacle depuis la page 25 , où la suggestion de la vengeance et du deuil se dévoile à travers ces vers :

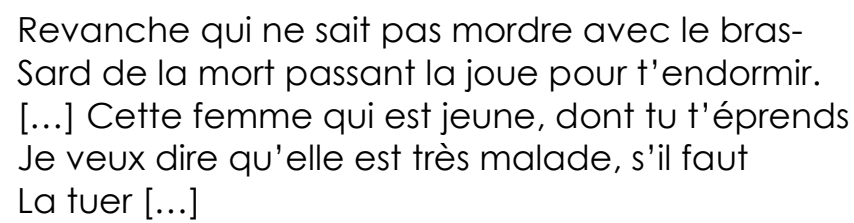

L'incipit "vengeance » de la page 26 est encore plus explicite car, il suggère le vocable de la guerre ("le carquois», "le harnais», les "chocs). Ce dispositif lexical pour "fai [re] mal» ( $v$. 6) signifie que le poète considère l'érotisme comme «la remarquable puissance d'énervement $\|^{8}$. Autrement dit, en prenant le relais, l'écriture poétique se présente comme cette nouvelle démence qui s'autorise toute extravagance dont la conclusion est la réalisation thanatique. La folie extrême conduit à la production d'un lexique hybride dans lequel de mystérieux bouts-de-mots se croisent, s'entremêlent, s'accouplent et s'entrechoquent sous l'effet des coups de butoir de l'acte sexuel. En effets, des termes comme "miséracle », "trifolium» "abardal», "lérou », entre autres, composent un répertoire de non-sens logique convoqué dans le juste dessein de contribuer à la "dé-figuration" du sens conventionnel frisant ainsi la démesure.

\subsection{Images de la démesure}

Roche, en photographe accompli, intègre à son spectacle érotique des images à profusion, des plus discordantes au plus rythmiques, en y adjoignant les plus affinées et les plus ludiques. On observe en effet, dans son style érotique, une prédilection pour la répétition à rythme régulier, le plus souvent saccadé, d'images de la dépravation. L'exemple des pages 32 et 33 se passe d'explication :

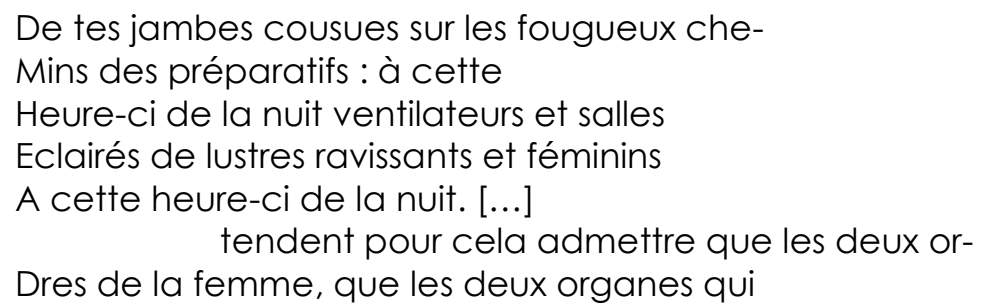

8 Roche, D. (2021). Éros énergumène, «Leçon sur la vacance poétique ». Paris : Gallimard, p. 16. 
Les lient comme aliments, comme semainiers.

[...] L'oléagineux,

Chez la dame à la douce chair, cette dame a

Le plus beau fessier de la région des solfatares

Des attributs physiques de la femme, la beauté et les parties du corps, sont évoquées au moyen des formes répétitives et rythmiques (" à cette heure-ci de la nuit ॥, «les deux ordres / organes », "la / cette dame ») dans le but d'un appel à la sensualité.

Dans la même veine, il convient de s'intéresser plus spécialement aux images intégrées au lexique de la navigation, comme à la page 28, pour leur charge symbolique essentiellement érotique :

Elle à la coupée serrant si fort ses mains
[...] ensevelie mêlée aux fleurs
De ce naufrage mais dans sa visite à l'enfer
[...] lumières des rayons orageux, la regardant s'
En aller l'éventail abaissé battant sa vulve
Battant sa bouche à la même seconde [...]
Et me parcourant lui font un instant détournée
Son désir malpropre d'après naufrage.
$\quad$ elle récite cette histoire de naufrage d'
Ensevelissement, c'est l'élan hors d'une solitude
Presque constante, où elle s'élance hors de ce
Bateau et court vers la cabane, déjà mouillée
Elle a même du lait pour me faire croire
me voicy je viens! Mais quelle certitude ?

Cet extrait semble rappeler Le bateau ivre rimbaldien` ou le Salut mallarméen, textes à l'intérieur desquels l'écriture poétique se conçoit systématiquement comme la restitution de la métaphore nautique. Mais dans le cas présent, Roche déploie les images de la navigation pour leur valeur hautement charnelle. Ainsi, la " coupée », qui est une ouverture pour la montée et la descente d'un navire, fait d'emblée figure d'une active chevauchée sexuelle. Le double emploi du participe présent «battant ", accompagnant les organes du plaisir sensuel ("vulve» et "bouche ») rappelle un autre battant, qui est un navire de guerre dont l'intérieur est agréable et aisé pour l'artillerie. Toutes ces images concourent merveilleusement à l'immensité du "naufrage » qui engloutit les amants et qui s'explique par la présence d'un univers thanatique ("visite à l'enfer», «Ensevelissement»). À l'évidence, l'orgie poétique rochienne s'inscrit élégamment dans une débauche sexuelle démentielle affectant fortement l'écriture dans tous ses aspects lexicaux et formels. D'une telle opinion presqu'exclusive, l'espoir d'une porte de sortie vers le spirituel est extrêmement résiduel.

\section{2. Éros énergumène comme un exposé de l'amour spirituel}

L'évidence du déploiement de l'amour charnel dans le corpus ne fait aucun doute, à telle enseigne que le " coït», envahissant en quelque sorte les lettres, prend le pas sur le récit lui-même. Cependant, force est de constater qu'une forme de spiritualité

\footnotetext{
${ }^{9}$ Arthur Rimbaud, Poésies, Une Saison en enfer, Illuminations. Paris, Gallimard, 1984, p. 34.
} 
accompagnant la matérialité de la chair se profile à travers l'écriture, qui se charge d'assumer sa part intellectuelle voire métaphysique.

\subsection{Posture d'intellectualité dans la poésie de Roche}

En prenant Kafka à témoin dans cette citation, Roche ambitionne sûrement d'évoquer le caractère davantage intellectuel de son Éros énergumène :

J'avais espéré satisfaire un peu mon amour pour elle en lui donnant mon bouquet. C'était complètement inutile. Cela n'est possible que par la littérature ou le coït. Je n'écris pas cela parce que je l'ignorais, mais parce qu'il est peut-être bon de mettre fréquemment les avertissements par écrit10.

Autant dire que la conception de l'activité sexuelle rochienne se compense par un équivalent intellectuel imbriqué dans l'acte de l'écriture. Le poète fait l'option, en effet, d'une cuisante expansion discursive dans le texte. En dépit de l'occurrence d'une syntaxe volontairement désorganisée, il n'est pas rare de rencontrer inopinément à l'intérieur du vers ou du groupe de vers une opinion, une explication, un commentaire ou une réplique dialogique. L'exemple du poème "Lectrice, tu frémis d'étonnement !... (pp. 65-67), fait état de cette constatation. D'un point de vue grammatical, la présence des deux points à l'intérieur des sous-titres conserve leur caractère explicatif ou définitionnel, mais constitue aussi une démarche présentative de théories propices à la démonstration ("Première mauvaise couleur: l'aise générale " / Deuxième mauvaise couleur: les idées claires /Troisième mauvaise couleur : les trois de l'escarpolette $»)$.

Le verbe « interpréter» de la «Première mauvaise couleur » souligne la tendance du poème à expliquer un fait:

$$
\begin{aligned}
& \text { [...] Ce qu'elle } \\
& \text { Me dit, ainsi je l'interprète en approchant de } \\
& \text { Cette terrasse immense, une ressemblance à s'y } \\
& \text { Méprendre, maintenant sans fond ni esprit na- } \\
& \text { turel, toujours à cours d'une bordure et de ton } \\
& \text { Aile, Théosophie... }
\end{aligned}
$$

L'obscurité de cette prétendue interprétation ne doit pas occulter l'idée de l'existence d'une démarche cogitative intégrée au texte poétique et qui se signale par la mention faite à la "Théosophie $\|^{11}$. La "deuxième mauvaise couleur " vient confirmer cette tentative de la réflexion intense à travers l'apparition de formes interrogatives, de propositions relatives entremêlées de prépositions :

quelle idée de vouloir goudronner ce que la
Vertu seule recèle ? L'histoire au Bec-d'Ambez ?
"Que la marque y restait toujours (...) "
-la seule qui fût permise enc
[...] Ma maîtresse et nous commençons bien avec
Lourdeur, avec ton âme, avec ton citron
[...] Cette fille dont nous parlons, par un seul pas
Quittant l'horizon ton bas-ventre énorme, et
Un autre pas lourdement menaçant l'héritière

\footnotetext{
10 Éros énergumène, p. 60.

11 La théosophie implique l'allusion à une somme de doctrines philosophico-religieuses que le poète ambitionne d'appliquer au développement intellectualisé du vers.
} 
La dernière "mauvaise couleur » se veut conclusive et persuasive au sujet des faits énoncés plus haut :
... Vois mon néant, dans le cours de toute
Cette anatomie de moi-même !...
De l'émouvoir encore, si les nerfs n'en
Sont intacts, etc. surpassant en beauté toutes
Les bergères morfondues melliflues le nom du serviteur et
Du soir m'empêche de prendre librement et la
Cause et l'essence pour d'autres roses qu'elle.

Cette mise en relief du néant (« Vois mon néant ») ressemble à l'objectif recherché par le poète, qui s' accorde une part de réflexion dans le corps du texte. Ici, à l'inverse du néant mallarméen, par exemple, qui se confond avec la négativité des mots, le néant rochien est énoncé simplement et présenté comme la finalité du langage qui dit le non-être des choses. La considération d'une branche d'intellectualité dans le cours du poème ouvre une lucarne sur la question de la spiritualité comme actualité métaphysique.

\subsection{Repères d'élévation dans Éros énergumène}

Le repère d'ascension le plus évident dans le poème semble être indiqué par l'intitulé de la page 71 («Elever cette conscience jusqu' au tendron ») :

Elever cette conscience jusqu'au tendron Et à l'entraille, aux ailes dressées en veux-tu L'emmener avec lui lui demanda si ce vœu était En accord avec celui de sa famille ? Le droit de. C'était un excellent point atteint, entre les Côtes 5 et 7, peut-être, sous le blanc? [...] Le printemps et l'automne c'est le même problème Qu'avec le veau tout blanc qui s'échappe et va Partout, semant son effroi blanc qui craque Nonnes et boiseries. Alors le vent était favorable E† s'allongèrent et demeurèrent là une grande Partie de l'été, écrivant de sanglantes raisons

Le motif de l'élévation dans le contexte baudelairien voire même symboliste est sensiblement biaisé chez le poète, ce qui fait qu'il se démarque. Chez Roche, en effet, "Elever [la] conscience " ne concerne certainement pas cette activité de l'esprit où la pensée doit se penser ${ }^{12}$ afin d'atteindre les "brumes" de l'absolu qui génèrent la pureté du mot poétique. Le spirituel poétique rochien se veut proche de ce qu'il convient d'appeler la nature des choses et des êtres en y incluant leur essence. C'est pourquoi l'ascension spirituelle $n$ 'atteint pas les hauteurs insondables, mais se limite au "tendron » frêle de la nature qui est, selon le poète, l' " excellent point atteint ". Les allusions au « blanc » rythmant le texte ont évidemment pour but d'évoquer une forme de pureté qui désacralise et dénature la pureté traditionnelle ou conventionnelle: «Partout, semant son effroi blanc qui craque / Nonnes et boiseries [...] ».

\footnotetext{
12 Nous paraphrasons Mallarmé qui, dans l'un de ses envois à son ami Cazalis, en 1867, écrivait ceci : « [...] Ma Pensée s'est pensée et est arrivée à une Conception Divine [...] $).$
} 
Tout se passe comme si, à l'intérieur de ce flux et reflux débordant de jouissance auquel fait allusion le vers 7 de la page 72 ("L'humidité amoureuse et la pluie incessante »), il se dessine systématiquement une forme féminine pure que l'écriture prend en charge : « la femme, l'adorable trouvaille qui te / Désigne (...) (p. 73). Mais de tout cela, il faut retenir que la nature constitue le point culminant de l'ascension rochienne. C'est en effet à travers la végétation, les paysages montagneux ou prairiaux, qu'affectionne béatement l'esprit du poète, que ce dernier atteint la plénitude:

[...] Près de l'onde et de l'

Os qui laboure les bois, légèrement au-dessus

De ce niveau de terre d'où paraissent se préci-

Piter avec omniscience les êtres marquants

Eł bouclés de notre plénitude. (p. 33)

Cette « remontée » basse, doit-on dire, à la hauteur du bas-astral, est suffisante pour le poète, qui désire perpétuer la « mélodie » des décombres de la sexualité :

[...] Son trépas

Résolu, la mélodie imaginant ma lente montée

Vers les prés de son existence [...] (p. 52)

Sur une parcelle qui est reboisée en pré

Epierrement élève défrichements paysages ruin...

[...] qui empêche que nous nommions ici

[...] Autre chose que [...]

La remontée dans le jus que tu m'exposes? (p. 75)

En somme, il s'agit ni plus ni moins de l'étalement de ce qu'il convient d'appeler la démence spirituelle, rythmée par le lexique de l'élévation, à l'intérieur d'un jeu érotique détraqué, évoqué et perpétué par l'écriture poétique.

\section{La démence réaliste à l'encontre du mythe surréaliste}

Il faut bien partir d'un fait (poétique, artistique et langagier s'entend) : à l'origine, se trouverait Mallarmé, considéré par Jean-Jacques Thomas comme l'un des acteurs essentiels de " la poésie extrême contemporaine ${ }^{13}$ ". Mais, la poésie de Denis Roche, aussi bien que celle de Gustave Roud, Francis Ponge et d' autres poètes, semble plutôt figurer sur la liste de la nouvelle poésie extrême inclassable. Éros énergumène est, en effet, du point de vue thématique, le prototype de la véritable folie poétique rendue par une écriture qui ne peut relever que de la névrose aussi bien langagière qu'érotique.

Certes, en se référant à l'ensemble du texte, il ressort qu' un certain destin surréaliste a pu être réservé à ce récit poétique qui mêle, de façon exceptionnelle, le primat de

\footnotetext{
13 Jean-Jacques Thomas, J.-J. (2009). Formulations: accessibilité et ostentation de la poésie extrême contemporaine. Formules, Actes du Colloque La Forme et l'informe dans la création moderne et contemporaine (Paris, Château de Cerisy-la-Salle, 11 au 18 juillet 2008). Paris: Nouvelle Imprimerie Laballery, 13, p. 97.

Cet article analyse des textes de poètes français et américains, en l'occurrence Mallarmé, Isidore Isou, Denis Roche, Jean-Marie Gleize, Charles Berstein, Bob Perelrnan, etc.
} 
l'image à l'extravagance linguistique. Cependant, il est aussi possible de noter une forme de mésestime du langage intérieur mutique du surréalisme auquel est préférée la lucidité insolite d'une écriture du mépris. L'analyse du poète Christian Prigent, à ce propos, contribue à accorder un point de départ à cette nouvelle poésie du méconnaissable :

[...] je suis allé voir plutôt du côté de ceux qui pervertissaient l'orthodoxie du surréalisme et en retournaient violemment les attendus théoriques: Artaud, Bataille, Ponge ; et que le «nouveau» a pris pour moi d'autres figures à partir de 1966 (les poètes beat américains), puis de 1968 (la mé-poésie de Denis Roche et la réflexion théorique de Tel Quel) ${ }^{14}$.

De cette aventure « mé-poétique » qui voit émerger le fameux Mécrit (1972), Denis Roche adopte définitivement la posture du poète dont la création langagière se veut avant tout "dé-création" de la poésie elle-même. Ainsi voit-on figurer dans les poèmes d'Éros énergumène un réalisme non seulement subverti mais également détrôné par la présence de formes linguistiques re-transfigurées. À l'évidence, les images tout comme le lexique et la syntaxe ne fonctionnent réellement dans le texte que sous une forme totalement désarticulée à tel point que le poète finira par réclamer, à cri et à cor, l'inadmissibilité de la poésie ${ }^{15}$. II s'agit d'admettre, d'une part, que Denis Roche s'insurge radicalement contre les théories surréalistes:

\begin{abstract}
A noter qu'une confusion au niveau de la théorie explique seule le débordement de bas lyrisme issu du surréalisme ; l'exploitation par celui-ci du fantastique inventé et du rêve réitéré (écriture soi-disant non contrôlée) servant d'alibi à une sorte de logorrhée de l'imagination supérieure (nostalgie de l'espèce de transcendance immédiate qu'on attribue avec tant d'empressement à la création poétique).
\end{abstract}

D'autre part, en adoptant la posture de rejet systématique de toute poésie admissible, c'est-à-dire concevable par sa marque normique, Roche ambitionne de hisser le texte poétique à une nouvelle sphère paroxystique où les mystérieux exposants moraux, affectifs ou sentimentaux sont écartés voire redimensionnés. C'est une démarcation notoire par rapport au lyrisme surréaliste qui, dans sa tentative d'investir les profondeurs inintelligibles de la psyché, se voit doté de la prétentieuse puissance suggestive de langage poétique. L'artifice inventif de Roche repose, comme il le dévoile en 1962, sur « la valeur émotionnelle des mots » :

D'autre part, j'écris toujours par séries de même inspiration et de même rythme. Car le rythme trouve sa véritable justification dans la chronologie, et c'est pour moi un souci constant de ne jamais faire un poème isolé. De là sans doute aussi le fait que j'ai rarement écrit des poèmes de plus d'une quinzaine de vers, craignant d'en étaler la densité au détriment de la valeur émotionnelle des mots ${ }^{16}$.

\footnotetext{
14 TXT et l'héritage surréaliste, entretien de Bénédicte Gorrillot avec Christian Prigent, http://www.polediteur.com, consulté le 2 février 2021.

15 En 1972, Denis Roche intitule une section de son Mécrit : « La Poésie est inadmissible ». Ce titre, quelque peu provocateur, sera retenu pour la réédition de ses œuvres poétiques complètes en 1995, aux éditions du Sevil.

16 Denis Roche, D. (1995). La Poésie est inadmissible (Avant-propos). Paris : Seuil.
} 
Chez lui, tout comme chez Mallarmé, l'invention poétique est possible lorsque l'initiative est octroyée aux mots de s'organiser rythmiquement dans les moindres allures du texte. En substance, il s'agit d'appréhender la poésie rochienne comme étant un jeu débordant de folie lexico-syntaxique, de délire démentiel, à travers lequel se profile une lucidité traduite par la volonté de "vengeance" scripturale. Cette vengeance inclue dans le poème est le prétexte visible et justifié de Denis Roche, qui s'engage à effectuer un travail poétique d'oblitération drastique du langage traditionnel.

\section{Conclusion}

L'intérêt que l'on peut accorder à Éros énergumène de Denis Roche est capital dans la mesure où, d'une part, la question de l'érotisme est analysée sous un double angle, à savoir : premièrement, il s'agit de la constatation effective d'un engorgement du texte par des formes lexico-syntaxiques et des images symboliques de la sexualité. Le dessein poétique de l'auteur s'inscrit, de ce fait, dans la perspective réaliste de monstration de détails vivants et impressionnants, afin que l'écriture, qui en est largement imprégnée, transcrive réellement les débordements et les dé-figurations de tous ordres. Secondement, la conception de la poésie nouvelle chez Roche, comme chez Gustave Roude, s'intègre à l'idée d'une transcendance intellectuelle et spirituelle dont le but est d'infléchir l'écriture vers un univers moins abscons que celui des symbolistes ou des surréalistes. La poésie de Roche, à travers Éros énergumène, se veut du point de vue formel la garantie de la nouvelle écriture de l'extrême démesure. Une telle option inventive concède à la fonction poétique une valeur supplémentaire de multiplication à l'infini de la création artistique, comme on la rencontre chez Raymond Queneau et Jacques Roubaud ${ }^{17}$.

\section{Bibliographie}

\section{Corpus}

RoCHE, D. (1995). La Poésie est inadmissible. OEuvres poétiques complètes. Paris : Seuil. ROCHE, D. (2021). Éros énergumène. Paris : Gallimard.

\section{Autres}

FRAZIER, F. (2007). Éros et Philia dans la pensée et la littérature grecques. Quelques pistes, d'Homère à Plutarque. Vita Latina, 177, pp. 31-44.

GORRILLOT B. TXT et l'héritage surréaliste, entretien avec Christian Prigent (en ligne), http://www.pol-editeur.com/ouverturepdf.php?file=005-txt-et-heritagesurrealiste.pdf [02/02/2021].

MAllarmé, S. (2001). Poésies. Paris : Gallimard.

MIRCEA, E. (1989). Mythes, rêves et mystères. Paris: Gallimard.

Queneau, R. (2006). Cent mille Milliards de Poèmes. Paris: Gallimard.

RIMBAUd, A. (1984). Poésies, Une Saison en enfer, Illuminations. Paris : Gallimard.

RoCHE, D. (1962). La Poésie est inadmissible. Paris : Sevil.

\footnotetext{
17 Deux poèmes majeurs de ces auteurs retiennent l'attention : Trente et un au Cube (1973) de Jacques Roubaud et Cent mille Milliards de Poèmes (1961) de Raymond Queneau. Un exercice visible de logique «illogique » et un déploiement formel à l'infini y sont appliqués vertement au langage poétique et proposés à la sagacité du lecteur.
} 
Rossı, M. (2010). Agapè ; l'amour sans limites. Paris : édition Robert Laffont. RoubaUd, J. (1973). Trente et un au Cube. Paris: Gallimard.

THOMAS, J.-J., (2009). Formulations : accessibilité et ostentation de la poésie extrême contemporaine. Formules, Actes du Colloque « La Forme et l'informe dans la création moderne et contemporaine " (Paris, Château de Cerisy-la-Salle, 11 au 18 juillet 2008). Paris : presses de la Nouvelle Imprimerie Laballery. 